\title{
Streamlining deep brain stimulation surgery by reversing the staging order
}

\author{
Craig G. van Horne, MD, PhD, ${ }^{1,2}$ Scott W. Vaughan, DO,' Carla Massari, MS, ${ }^{1}$ \\ Michael Bennett, PA-C, MS, ${ }^{1}$ Wissam S. Z. Asfahani, MD, ${ }^{2}$ Jorge E. Quintero, $\mathrm{PhD},{ }^{2}$ and \\ Greg A. Gerhardt, PhD²
}

\begin{abstract}
'Department of Neurosurgery, St. Elizabeth's Medical Center, Brighton, Massachusetts; and 'Department of Neurosurgery, University of Kentucky, Lexington, Kentucky
\end{abstract}

\begin{abstract}
Deep brain stimulation (DBS) is approved for several clinical indications; however, the sequencing of DBS surgery and the timeline for implementing stimulation therapy are not standardized. In over 140 cases so far, the authors have reversed the sequencing for staged implantation of DBS systems that was conducive to minimizing patient anxiety and discomfort while providing the opportunity to shorten the time between implantation and programming for therapeutic management of symptoms. Stage I was performed with the patient under general anesthesia and consisted of implantation of the pulse generator and lead extensions and placement of the bur holes. Stage II was completed 1-7 days later, using only local anesthesia, and included stereotactic frame-based microelectrode recordings, semi-microstimulation and macrostimulation, and testing and placement of the stimulating electrodes. Stage I lasted approximately 90 minutes, whereas Stage II lasted approximately 230 minutes. All patients tolerated the procedures and received a complete implanted system. Deep brain stimulation therapy was typically initiated on the same day as lead implantation. When sequencing was reversed and bur holes were placed during the first stage while a patient was under general anesthesia, the patient was able to tolerate the second awake stage and was able to begin stimulation therapy within 48 hours of the second stage.
\end{abstract}

http://thejns.org/doi/abs/10.3171/2014.9.JNS14619

KEY WORDS deep brain stimulation; neuronavigation; anesthesia; functional neurosurgery

$\mathrm{D}$ EEP brain stimulation (DBS) is FDA approved for several clinical indications, including Parkinson's disease, essential tremor, and dystonia. Additional investigational applications, such as obsessive-compulsive disorder, dementia, depression, and obesity, are on the horizon for widespread clinical practice., ${ }^{3,59}$ Appropriate DBS therapy involves proper patient evaluation and selection; surgical intervention; and device programming initiation, adjustment, and readjustment. Currently, there is no standard protocol for the timing, staging, or sequencing of these procedures. Although some neurosurgeons elect to perform all components of the surgery in 1 day, a recent survey indicates that $87 \%$ of North American and $34 \%$ of European DBS surgeries are performed in multiday stages. ${ }^{1}$ Deep brain stimulation system implantations are performed in stages, in part to decrease the patient's time in surgery and, to some extent in the US, for reimbursement considerations. ${ }^{8}$ Staged procedures can be performed in 2,3 , or as many as 4 separate surgeries. ${ }^{1,2,6-8}$ Thus, in the most protracted scenario, patients undergo 2 surgeries while under general anesthesia, 2 stereotactic frame placements, and 2 awake surgeries. Meanwhile, the effi-

ABBREVIATIONS CRW = Cosman-Roberts-Wells; DBS = deep brain stimulation. SUBMITTED April 2, 2014. ACCEPTED September 10, 2014.

INCLUDE WHEN CITING Published online March 6, 2015; DOI: 10.3171/2014.9.JNS14619.

DISCLOSURE The authors report no conflict of interest concerning the materials or methods used in this study or the findings specified in this paper. Dr. van Horne is a consultant for Medtronic. Support was provided in part by University of Kentucky start-up funds (C.vH.) and the National Center for Advancing Translational Sciences (Grant No. UL1TR000117). The content is solely the responsibility of the authors and does not necessarily represent the official views of the NIH. 
cacy of the procedure and therapy may not be determined, sometimes for weeks after surgery, until the stimulator is activated and programming is initiated. The most common feature of the staging is the sequencing, in which the awake procedure for DBS lead placement is performed first, followed days to weeks later by pulse generator implantation under general anesthesia. Although many factors play a role in the specifics of the process, the precise sequencing and timing are ultimately determined by the attending neurosurgeon.

Based on feedback from patients and clinicians, we critically reviewed the technical aspects of the complete procedure and designed several changes to the process. Our major goals focused on improving the patient experience and optimizing opportunities for ensuring successful DBS lead placement. The most substantial change was the reordering of the typical sequence of the two surgeries; that is, instead of performing the awake procedure and DBS lead placement followed by pulse generator placement under general anesthesia, we reversed the stages. Implementing this reversal required novel approaches to some of the surgical techniques. The specific details of the modifications and implications in a series of patients who underwent DBS surgery at our institutions are presented in this paper.

\section{Methods \\ Patients}

Over 140 patients to date (Table 1), ranging in age from 21 to 81 years, have been treated via reversed staging DBS surgery for Parkinson's disease, dystonia, essential tremor, or tremor due to multiple sclerosis. Each patient was evaluated by a movement disorder neurology team and was deemed a good candidate for DBS therapy in accordance with standard criteria. ${ }^{4}$ Reversed staging for DBS has now been adapted at our institution as the standard of care.

\section{Materials}

Soletra, Kinetra, or Activa (Medtronic Inc.) pulse generators were unilaterally or bilaterally implanted in conjunction with lead extensions (model number 7482/7483, Medtronic Inc.) and stimulating electrode leads (model number 3387, Medtronic Inc.).

Although for the first several patients we initially used the Navigus StimLoc hardware (Medtronic Inc.) to stabilize and secure the stimulating lead at the bur hole, we subsequently developed a low-profile method that utilizes the following: Hydroset (Stryker), a rapid-setting calcium phosphate cement; a 2-hole titanium adaptation plate

\section{TABLE 1. Diagnoses in patients who underwent DBS surgery,} across 2 institutions

\begin{tabular}{lcc}
\hline \multicolumn{1}{c}{ Disease } & Institution 1 & Institution 2 \\
\hline Parkinson's disease & 44 & 48 \\
\hline Essential tremor & 8 & 33 \\
\hline Dystonia & 2 & 6 \\
\hline Multiple sclerosis & 1 & 0 \\
\hline Total & 55 & 87 \\
\hline
\end{tabular}

(Synthes) along with 2 titanium cranial screws; and the lead end of the lead-extension connector boot (Medtronic Inc.) or, alternatively, a 1-cm segment of a large ventricular catheter (82-1735, Codman).

Brainlab intraoperative workstation and neuronavigation software (VectorVision2 and IPlanStereotaxy) were used for frameless stereotactic planning and targeting for bur hole placements as well as for frame-based targeting of the microelectrode recording trajectories. The CosmanRoberts-Wells (CRW) stereotactic frame (Integra) was used for microelectrode and DBS lead targeting. Intraoperative microelectrode recordings were performed with the Alpha Omega system (Israel), Leadpoint (Medtronic Inc.), or Guideline 4000LP+ (FHC Inc.) workstations using microelectrodes (FHC Inc.).

\section{Description of Surgical Technique \\ Stage I}

Our first-stage surgery is a preparatory procedure performed with the patient under general anesthesia. This stage consists of placing the pulse generator in a subclavicular pocket, tunneling and connecting lead extensions to the pulse generator, and creating unilateral or bilateral frontal bur holes.

Several days or weeks before surgery, patients undergo outpatient noncontrast MRI. We use preoperative neuronavigational planning to determine the entry point for each lead, allowing appropriate placement for the skin incisions and the bur hole locations. Using neuronavigation software, we select targets for the DBS leads according to standard criteria. Corresponding trajectories are chosen and typically course close to the coronal suture, through a cortical gyrus to avoid the sulcal vasculature, and lateral to the lateral ventricle. The trajectory of the lead is surface matched using Z-touch and Softouch (Brainlab) and marked as the center at which the bur hole should be formed.

An incision is then created in the postauricular area just above and behind the mastoid to facilitate tunneling. The extension leads $(60 \mathrm{~cm})$ are tunneled from the infraclavicular incision to the postauricular incision using the provided tunneler. The distal ends of the extension leads are then tunneled under the galea to the frontal incisions, capped, and tucked in the subgaleal space, where they will be accessible during the Stage II surgery (Fig. 1). All incisions are closed and sterile bandages are applied.

A variation for the tunneling involves the use of a single $10-\mathrm{Fr}(3.3 \mathrm{~mm})$ Rob-Nel red rubber catheter, cut in $2 \mathrm{seg}-$ ments and trimmed to length, as a conduit system from the bur hole regions to the postauricular connector incision to pass the proximal end of the DBS leads under the scalp to the connector end of the lead extensions. We label the conduits at the site of the lead extension to permit accurate connection to the proximal DBS leads in Stage II. This technique allows the use of shorter extension leads and leaves extension lead connectors in the retroauricular area.

Stage I is scheduled as an outpatient procedure. Although most patients go home the same day, some patients may be admitted for 24-hour observation and then discharged the following morning, depending on clinical indications. 


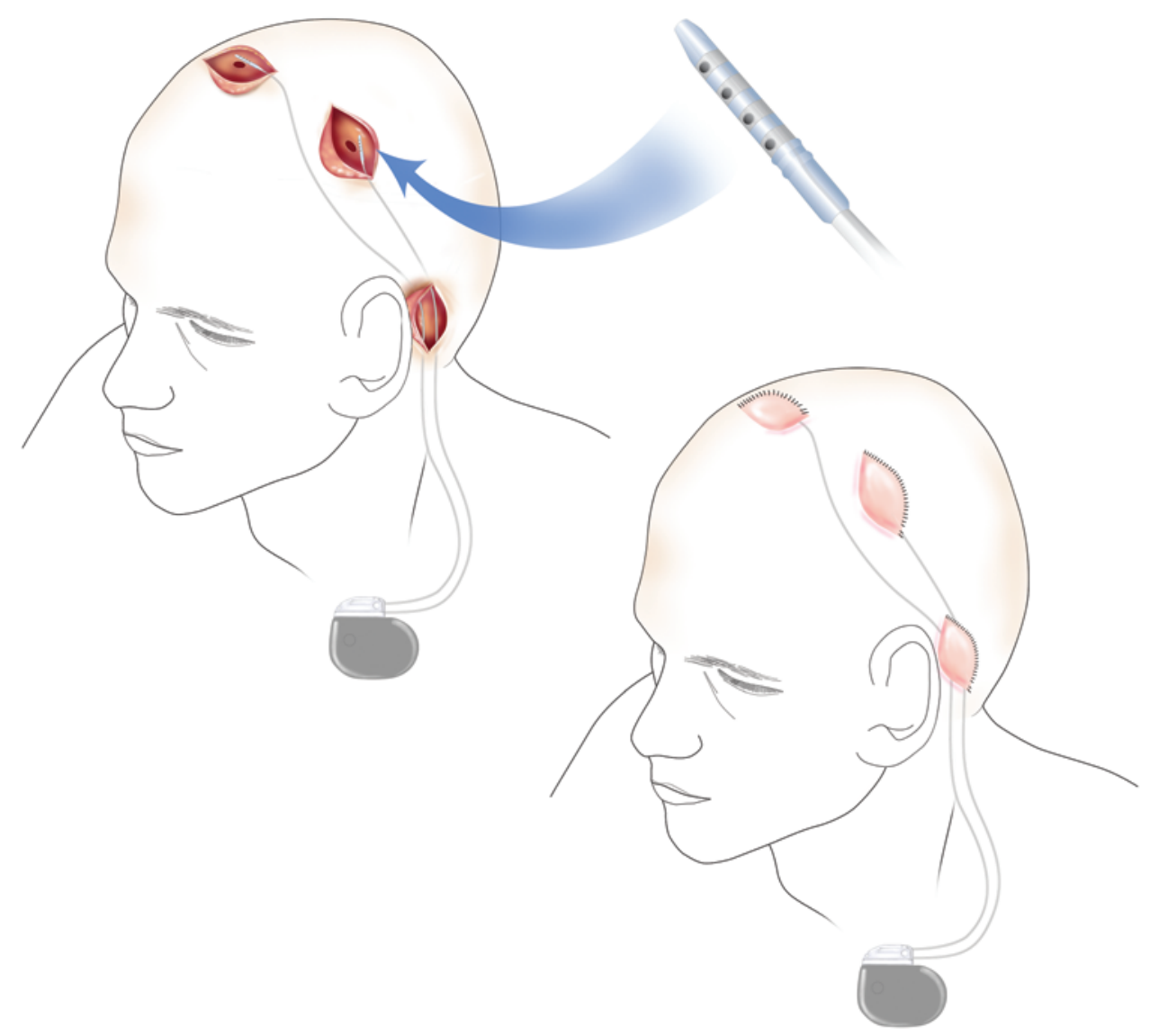

FIG. 1. Stage I surgery is performed with the patient under general anesthesia and involves the placement of bilateral frontal bur holes, subgaleal tunneling of the lead extensions to the coronal incision sites, and implantation of the pulse generator. Copyright University of Kentucky Analytics and Technologies. Published with permission. Figure is available in color online only.

\section{Stage II}

The second stage is performed within 3-7 days following the Stage I procedure. The second stage has been performed the following day in some patients for whom long distance travel was a significant factor. This stage focuses on the placement of DBS leads into the desired targets and is performed after applying a local anesthetic to allow microelectrode recording, test stimulation of DBS leads, and patient examination.

On the morning of surgery in the preoperative area, a CRW frame is placed on the patient after a local anesthetic has been administered. A CT scan with the CRW localizer is obtained and fused with the MRI targeting data using the surgical planning software. The stereotactic coordinates of the targets are obtained and confirmed from the neuronavigation software, and the trajectory is adjusted, if necessary, to pass through the bur hole and a cortical gyrus while avoiding the sulcal vasculature and ventricle walls.

Once in the operating room, patients receive intravenous Tylenol as an analgesic and, if needed, a Cardene drip for blood pressure control. No sedation is given. The previously created frontal incision is reopened after the injection of a local anesthetic, the dura mater is opened, and microelectrode recordings are obtained through the targeted trajectory. Microstimulation is performed, and the patient is tested for clinical benefit and stimulation side effects. When clinically satisfactory results have been obtained, a DBS lead is inserted into the appropriate area and tested for impedance performance as well as for clinical benefit and side effect profiling. After a satisfactory assessment, the lead is then secured and connected to the lead extension. As the proximal end of the extension leads are pretunneled to the frontal incision sites during Stage I, the extensions are easily found in the subgaleal space. To secure the lead, a small piece of Durepair (Medtronic, Inc.) is cut to the size of the bur hole and is placed around the lead on top of the dura. The bur hole is filled with Hydroset bone cement, which is then allowed to harden (approximately 3 minutes). The DBS lead is then secured to the underlying skull using a pre-bent 2-hole titanium plate and screws. A small piece of ventricular catheter or the lead end of the lead-extension connector boot is cut and opened lengthwise to serve as a protective sleeve around the lead at the site of contact with the titanium plate (Fig. 2). In our initial cases, we used red rubber conduits that were placed in the subgaleal space running from each bur hole to the connector incision. The conduits were then used to guide the distal end of the secured DBS leads from the bur holes to the connectors during Stage II, obviating the need for tunneling while the patient is awake. Conduits 


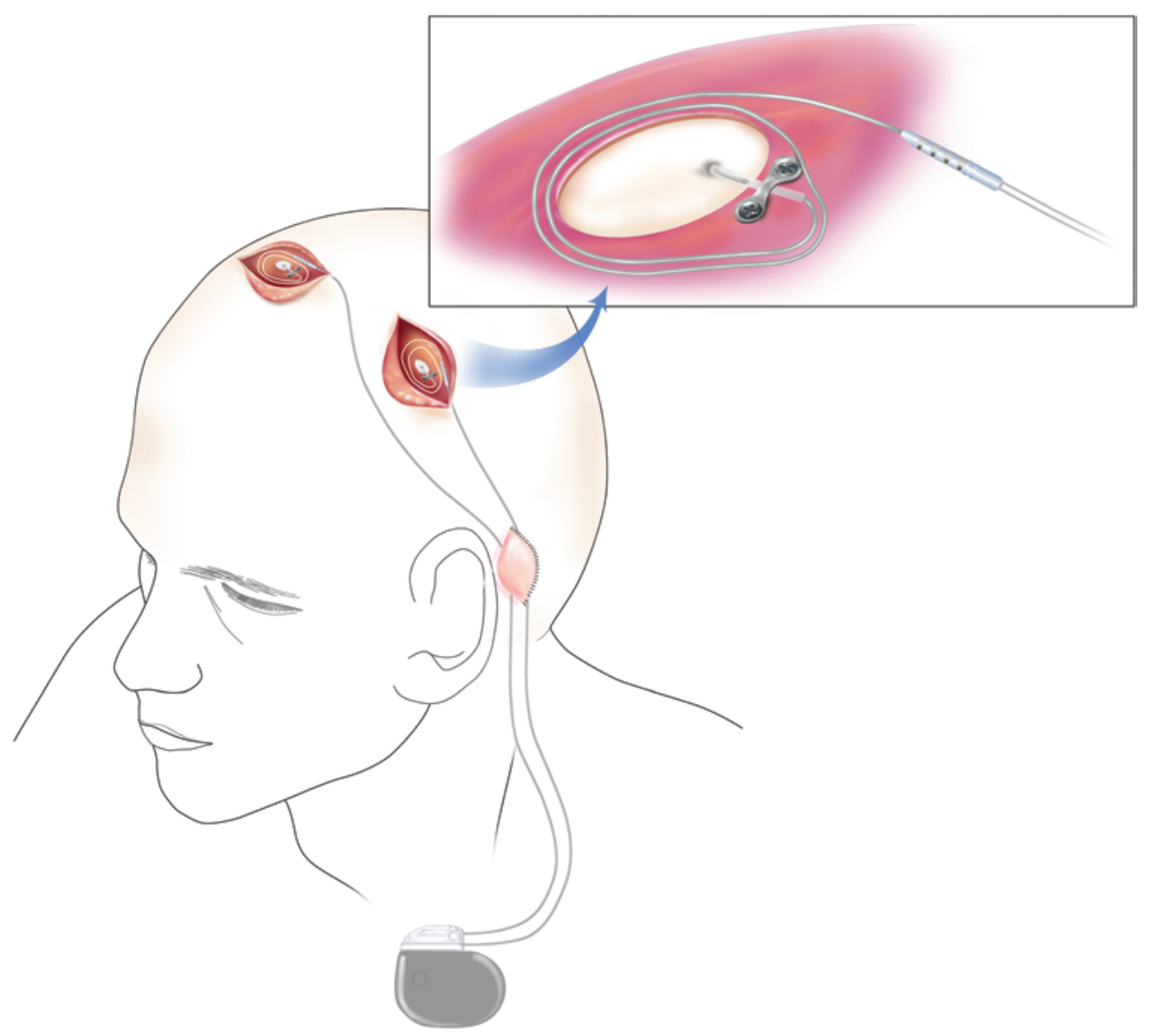

FIG. 2. In the Stage II surgery, while the patient is awake, the DBS distal leads are positioned at the desired target and secured to create a lower profile. The proximal portions of the leads are connected to the lead extensions implanted in Stage I. In turn, the use of conscious sedation is avoided. Copyright University of Kentucky Analytics and Technologies. Published with permission. Figure is available in color online only.

were removed prior to connecting the proximal end of the DBS lead to the lead extensions.

At the conclusion of Stage II, the patient is removed from the CRW frame, transported to the postanesthesia care unit for recovery, and admitted overnight for observation. Postoperative CT scanning is performed to verify targeting and assess the possibility of pneumocephalus or hemorrhage.

\section{Results}

For the initial 31 patients, we noted that the Stage I surgery lasted approximately 90 minutes from incision to closing, whereas Stage II lasted approximately $230 \mathrm{~min}-$ utes. The timing of subsequent surgeries agreed with these measurements. Nearly all patients were discharged within 48 hours of implantation.

In our series, none of the attempted electrode placements had to be aborted because of technical difficulties or patient intolerability, and complete systems were implanted in all patients. We found no infections of the red rubber catheters or scarring around the proximal connector that interfered with placement of the DBS electrode. One patient developed a liver abscess, discovered on preoperative evaluation before his second-stage surgery. Thus, his sec- ond stage was postponed, and he was treated for the infection. As the infection was remote from the hardware, his system remained in place, and bilateral stimulating electrodes were successfully implanted several months later.

Nearly all patients underwent initial programming of their stimulator systems during the postoperative hospital stay. Initial configurations were chosen based on intraoperative results and clinical tolerance. Medications were adjusted according to clinical need. We have not observed any significant adverse events related to early stimulation.

\section{Discussion}

The conventional approach to DBS surgery involves microelectrode recordings and placement of the DBS leads using conscious sedation and local anesthesia during the first stage, followed by a second stage in which the pulse generator and lead extensions are implanted while the patient is under general anesthesia. Traditionally, staging has depended on surgeon preference, the surgical center, and the number of devices implanted. Rather than making a claim that our reversed-stages procedural modification is superior to the conventional staging approach, we have outlined details on the new approach to provide an additional option for surgical DBS teams to consider. 
One potential criticism of the technique is that the pulse generator and lead extensions are implanted before the stimulating leads are placed, and a problem could arise that would prevent final placement of the leads, rendering the implanted system nonfunctional. We have found that this risk is very low given that we have used this staging sequence in over 140 cases without having to abandon placement of the stimulating electrodes. Additionally, advances in target visualization, target computation, and electrophysiological analysis have significantly improved the success rate of intraoperative target localization and stimulation electrode placement. Therefore, we do not believe that there is a substantial downside to implanting the pulse generator and lead extensions first. Conversely, we have encountered several patients from other centers at our clinic who have decided to not undergo the second stimulating electrode implantation, citing the anxiety and discomfort created by the experience of the bur hole drilling.

In the protocol described here, patients undergo the targeting MRI without a frame before the preoperative visit. Acquiring images in advance of the preoperative visit allows ample time for review to confirm image quality and to determine that there are no extenuating anatomical or pathological circumstances. In addition, having the MRI studies at that point allows adequate surgical planning for target location and trajectory placement. Furthermore, on the day of the Stage I surgery, only a CT scan is required after frame placement, which facilitates operating room workflow and creates less stress for the patient.

Patients also undergo bur hole placement under general anesthesia during Stage I, which has several advantages. First, patients do not have to experience drilling during the awake portion of the procedure. During conventionally staged procedures, a short-acting anesthetic or conscious sedation is used and then reversed after the bur hole is placed. Nevertheless, several patients treated at our clinic who have undergone the conventional techniques clearly recall the experience and described it as unpleasant and anxiety provoking. In addition, some patients, especially those with Parkinson's disease who are off their medications, may have difficulty tolerating the experience and the effects of sedation. Thus, with our protocol, patients can undergo microelectrode recordings without any sedation and are more likely to be fully awake and interactive during the clinical testing portion of the procedure. The lack of sedative medications also decreases the possibility for interference with the electrophysiological recordings throughout the targeted areas. We have also observed that our patients are more likely to make it through a bilateral procedure with less fatigue and more mental clarity.

With respect to operating room workflow, the timing we observed for each of our stages-approximately 90 minutes for Stage I and 230 minutes for Stage II-is in accordance with the reported times for these bilateral procedures under conventional conditions. ${ }^{1}$ Unilateral procedures took considerably less time. All patients, either with unilateral or with bilateral implantations, received implants in 2 stages; that is, none of the patients selected for bilateral implantation underwent 2 separate unilateral procedures.
Our current protocol allows patients to have DBS systems implanted and completely functional within 3-5 days. This is the shortest timespan possible without performing the surgeries in a single inpatient setting. Although patients can tolerate a shorter gap between surgeries, as when they are performed in one hospital admission, US hospitals lose a significant amount of money because of reimbursement restrictions set forth by coding regulations. Conversely, in most states, there is a financial advantage to staging the stimulator implantation surgeries separately by more than 30 days. Under these conditions, the second stimulator implant is considered a stand-alone procedure rather than a staged procedure or an add-on procedure.

In summary, we believe that the approach outlined here has several potential advantages that could benefit the patients as well as the surgical teams and is worth considering as an alternative to the traditional staging paradigm. We have found that it is particularly beneficial in situations in which patients need to be fully awake and interactive to ensure accurate targeting and the avoidance of stimulation side effects.

\section{Conclusions}

We present a DBS surgical protocol in which the typical staging sequence is reversed: the pulse generator and lead extensions are implanted during the first stage, and the microelectrode recordings and stimulating lead placements are performed during the second stage. We take advantage of this sequence by creating the bur holes during the first stage while the patient is under general anesthesia. One of the main benefits of this particular surgical staging sequence is the minimization of patient discomfort and anxiety by eliminating any awareness of the bur hole experience. Additionally, we feel we have the ability to devote more time to microelectrode recordings and DBS lead placement given that the bur holes have already been placed, and the patients are free of any effects from sedatives. Finally, patients have the opportunity to begin stimulation therapy in a more streamlined fashion after having their complete system implanted within 3-5 days.

\section{Acknowledgment}

We thank Tom Dolan at the UK Academic Technology Group for the illustrations.

\section{References}

1. Abosch A, Timmermann L, Bartley S, Rietkerk HG, Whiting D, Connolly PJ, et al: An international survey of deep brain stimulation procedural steps. Stereotact Funct Neurosurg 91:1-11, 2013

2. Bejjani BP, Dormont D, Pidoux B, Yelnik J, Damier P, Arnulf I, et al: Bilateral subthalamic stimulation for Parkinson's disease by using three-dimensional stereotactic magnetic resonance imaging and electrophysiological guidance. J Neurosurg 92:615-625, 2000

3. Halpern CH, Torres N, Hurtig HI, Wolf JA, Stephen J, Oh MY, et al: Expanding applications of deep brain stimulation: a potential therapeutic role in obesity and addiction management. Acta Neurochir (Wien) 153:2293-2306, 2011

4. Lang AE, Widner H: Deep brain stimulation for Parkinson's 
disease: patient selection and evaluation. Mov Disord 17 Suppl 3:S94-S101, 2002

5. Lozano AM, Lipsman N: Probing and regulating dysfunctional circuits using deep brain stimulation. Neuron 77:406424, 2013

6. Rezai AR, Kopell BH, Gross RE, Vitek JL, Sharan AD, Limousin P, et al: Deep brain stimulation for Parkinson's disease: surgical issues. Mov Disord 21 (Suppl 14):S197-S218, 2006

7. Shrivastava RK, Germano IM: Deep brain stimulation for the treatment of Parkinson's disease. Contemp Neurosurg 23:1-9, 2001

8. Sierens DK, Kutz S, Pilitsis JG, Bakay RAE: Stereotactic surgery with microelectrode recordings, in Bakay RAE (ed): Movement Disorder Surgery: The Essentials. New York: Thieme, 2009, pp 83-114

9. Yeremeyeva E, Taghva A, Rezai AR: Seeking new solutions: stimulation of diseased circuits in depression and other neurobehavioral disorders. Clin Neurosurg 59:44-49, 2012

\section{Author Contributions}

Conception and design: van Horne. Drafting the article: van Horne, Vaughan, Massari, Bennett. Critically revising the article: van Horne, Asfahani, Quintero, Gerhardt. Reviewed submitted version of manuscript: all authors. Approved the final version of the manuscript on behalf of all authors: van Horne. Administrative/technical/ material support: Massari. Study supervision: van Horne.

\section{Correspondence}

Craig G. van Horne, Department of Neurosurgery, University of Kentucky, 800 Rose St., Lexington, KY 40536. email: craigvanhorne@uky.edu. 